\title{
CSF1R inhibition by a small molecule inhibitor affects hematopoiesis and the function of macrophages.
}

Fengyang Lei MD, PhD, ${ }^{1,2,3}$ Naiwen Cui, $\mathrm{PhD},{ }^{4}$ Chengxin Zhou $\mathrm{PhD},{ }^{1,2,3}$ James Chodosh, MD, MPH, ${ }^{1,2,3}$ Demetrios G. Vavvas MD, PhD, ${ }^{1,5}$ Eleftherios I. Paschalis MSc, PhD, ${ }^{1,2,3}$

${ }^{1}$ Massachusetts Eye and Ear, Department of Ophthalmology, Harvard Medical School, Boston, MA

${ }^{2}$ Boston Keratoprosthesis Laboratory, Harvard Medical School, Boston, MA

${ }^{3}$ Disruptive Technology Laboratory, Massachusetts Eye and Ear, Department of Ophthalmology, Harvard Medical School, Boston, MA

${ }^{4}$ John A. Paulson School of Engineering and Applied Sciences, Harvard University, Cambridge, MA 02138, USA

${ }^{5}$ Angiogenesis Laboratory, Massachusetts Eye and Ear, Department of Ophthalmology, Harvard Medical School, Boston, MA

Corresponding author and reprints: Eleftherios I. Paschalis, Department of Ophthalmology, Boston Keratoprosthesis Laboratory, Massachusetts Eye and Ear and Schepens Eye Research Institute, Harvard Medical School, Boston, MA 02114, USA;

e-mail: eleftherios_paschalis@meei.harvard.edu

Conflict of Interest: The authors have declared that no conflict of interest exists.

Key Words: Eye; neuroglia, microglia; macrophage; remodeling, CSF1R.

Running title: Long-term effect of CSF1R inhibition on hematopoiesis. 


\begin{abstract}
Colony-stimulating factor 1 receptor (CSF1R) inhibition has been proposed as a method for microglia depletion, with the assumption that it does not affect peripheral immune cells. Here, we show that CSF1R inhibition by PLX5622 indeed affects the myeloid and lymphoid compartments, causes long-term changes in bone marrow-derived macrophages by suppressing their IL-1 $\beta$, CD68 and phagocytosis, but not CD208, following exposure to endotoxin, and also reduces the population of tissue resident macrophages of peritoneum, lung, liver, but not spleen. Thus, small molecule CSF1R inhibition is not restricted to microglia only, but rather causes strong effects on circulating and tissue macrophages that perdure long after cessation of the treatment. Given that peripheral monocytes repopulate the CNS after CSF1R inhibition, these changes may have practical implications on relevant experimental data.
\end{abstract}

Ibody

Colony stimulating factor 1 receptor (CSF1R) inhibition has been proposed as a specific method for microglia that does not affect peripheral immune cells(1-4). However, this claim has been based solely on cell count measurements of blood monocytes and evaluation of blood brain barrier rather than direct assessment of cellular subtypes and their function. Also, recent data have shown an effect on liver and lung tissue resident macrophage $(5,6)$ Given that peripheral monocytes have been shown to participate in CNS disease via both infiltration and repopulation of neuroglia following microglia depletion(4, 7-12), it is important to determine if CSF1R inhibition causes functional changes in peripheral immune cells that can become part of the CNS(3, 4, 12-16).

Here we show that, contrary to the accepted notion(1, 13), PLX5622, a commonly used small molecule CSF1R inhibitor(10, 12, 13), does not affect only microglia but also leads to long-term changes in the myeloid and lymphoid compartments of the bone marrow, spleen and blood by suppressing CCR2 ${ }^{+}$monocyte progenitor cells, $\mathrm{CX} 3 \mathrm{CR} 1^{+}$bone marrow-derived macrophages (BMDM), CD117 ${ }^{+}\left(\mathrm{C}-\mathrm{KIT}^{+}\right)$hematopoietic progenitor cells, F4/80 ${ }^{+}, \mathrm{MerTK}^{+}$and CD $34^{+}$hematopoietic stem cells (fig. 1 A-G). Most importantly, these cell populations either do not recover or rebound after cessation of CSF1R inhibition, with the exception of CD45 $5^{+}$

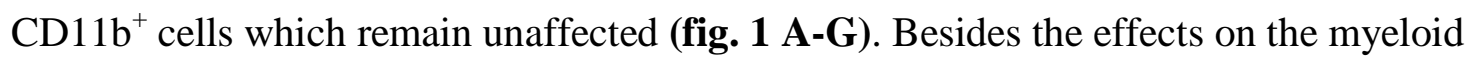
compartment, CSF1R inhibition also alters the lymphoid compartment of the bone marrow by suppressing T-cells $\left(\mathrm{CD}^{+}, \mathrm{CD} 4^{+}\right.$, and $\mathrm{CD} 8^{+}$), (fig. 1 G) and by upregulating $\mathrm{CD} 19^{+} \mathrm{B}$ cells (fig. 1 G). Cessation of CSF1R inhibition causes rebound of some, but not all, lymphoid cells (fig. 1 G).

In addition to the effects on bone marrow cells, CSF1R inhibition also suppresses splenic $\mathrm{CX} 3 \mathrm{CR} 1^{+}$cells and this inhibition persist for at least 3 weeks after cessation of treatment (fig. 1 H). Moreover, splenic $\mathrm{CD}^{+} \mathrm{T}$ cells (primarily $\mathrm{CD} 8^{+}$) become suppressed, whereas $\mathrm{CD} 19^{+} \mathrm{B}$ cells are not affected by the inhibitor (fig. 1 H).

Likewise, CSF1R inhibition suppresses circulating CX3CR $1^{+}, \mathrm{CD}_{115^{+}}, \mathrm{F} 4 / 80^{+}$and MerTK ${ }^{+}$blood cells with no immediate effect on the lymphoid $\mathrm{CD}^{+}$and $\mathrm{CD}^{+}$populations (fig. 
1 I). Cessation of CSF1R inhibitor causes rebound of the $\mathrm{CX} 3 \mathrm{CR} 1^{+}, \mathrm{F} 4 / 80^{+}$and $\mathrm{MerTK}^{+}$, but not $\mathrm{CD} 115^{+}$blood cells, and leads to delayed suppression of $\mathrm{CD} 19^{+}, \mathrm{CD}^{+}$and $\mathrm{CD} 4^{+}$lymphoid cell in the circulation (fig. 1 I).

CSF1R inhibition also suppresses the proliferation of bone marrow and spleen macrophages (fig. 2 A-D), and impairs the function of bone marrow-derived macrophages (BMDM) for the long-term. In fact, 3 weeks after cessation of CSF1R inhibition, BMDMs display reduced IL-1 $\beta$ expression in response to endotoxin (fig. 2 E, F), diminished phagocytosis (fig. 2 E-G), reduced CD68, but not CD206 expression (fig. 2 I-L), and suppressed CD1 15 labeling (fig. 2 M-N). Moreover, CSF1R inhibition reduces the number of tissue resident $\mathrm{CX}_{3} \mathrm{CR} 1^{+}$femur, CD11a ${ }^{+}$lung, CD102 $2^{+}$peritoneum, MHC-II ${ }^{\mathrm{hi} / \mathrm{lo}}$ liver, but not $\mathrm{CD}_{106^{+}}$spleen macrophages, while consistently suppresses bone marrow-derived CX3CR $1^{+/ E G F P}$ macrophages in these tissues (fig. 2 O).

Previous studies have suggested that microglia depletion by CSF1R inhibition can either promote or exacerbate neurodegeneration $(4,12,17)$. This puzzling and contradictory results may not only be due to the differing role of microglia in various disease models but also due to the varying relative contribution of peripheral and circulating macrophages on disease phenotype. Our study demonstrates that CSF1R inhibition affects both the circulating and tissue resident macrophages of various tissue which may explain in part the reason for the discrepancy in recent investigations.

Considering that BMDMs permanently engraft into diseased CNS tissue(10, 12), this work suggests that small molecule inhibition is not restricted to microglia but additionally affects the turnover and function of bone marrow-derived, circulating and tissue resident macrophages. These effects perdure long after cessation of the treatment and has implications in the interpretation of relevant experimental data.

\section{Materials and Methods}

Mouse model: All animal experiments were performed in accordance with the Association for Research in Vision and Ophthalmology Statement for the Use of Animals in Ophthalmic and Vision Research, and the National Institutes of Health (NIH) Guidance for the Care and Use of Laboratory Animals. This study was approved by the Mass. Eye and Ear Animal Care Committee. Mice at 6-12 months old were used: C57BL/6J (Stock\#: 000664), B6.129(Cg)Ccr2tm2.11fc/J (Stock\#: 017586) and B6.129P- Cx3cr1tm1Litt/J (Stock\#: 005582) Jackson Laboratory. CCR2 ${ }^{\mathrm{RFP} /+}:: \mathrm{CX} 3 \mathrm{CR} 1^{\mathrm{EGFP} /+}$ generated by crossing B6.129(Cg)-Ccr2tm2.11fc/J with B6.129P- Cx3cr1tm1Litt/J. CX3CR $1^{\mathrm{EGFP} /+}$ generated by crossing male B6.129P$\mathrm{Cx} 3 \mathrm{cr} 1 \mathrm{tm} 1 \mathrm{Litt} / \mathrm{J}$ with female C57BL/6J. Mice were bred in house. Microglia depletion was performed by chow administration for 3 weeks of PLX5622 (Plexxikon Inc., Berkeley, CA). Flow cytometry, and ex vivo BMDM evaluation was performed as previously described(10, 12). Blood cells was collected by cardiac puncture and centrifugation. Peritoneum, lung, liver, spleen and femur resident macrophage were evaluated using appropriate markers $(18,19)$ in BMT CX3CR $1^{+/ E G F P}$ reporter mice following 3-weeks exposure to PLX5622. 
Flow cytometry markers: Bone marrow and spleen cells from $\mathrm{CX} 3 \mathrm{CR} 1^{+/ \mathrm{GFP}}$ and $\mathrm{CX} 3 \mathrm{CR} 1^{+/ \mathrm{EGFP}}:: \mathrm{CCR} 2^{+/ \mathrm{RFP}}$ reporter mice were blocked with CD16/32 (Clone: $2.4 \mathrm{G} 2$ ), analyzed with IL-1 $\beta$ (Clone: NJTEN3), Lyve1 (Clone: ALY7) eBiosciences (San Diego, CA); CD45 (Clone: 104), CD11b (Clone: M1/70), CD11c (Clone: N418), CD3 (Clone: 17A2), CD4 (Clone: GK1.5), CD8 (Clone: 53-5.8), CD19 (Clone: 6D5), CD117 (Clone:2B8), CD34 (Clone: HM34), CD115 (Clone: AFS98), CD68 (Clone: FA-11), CD206 (Clone: C068C2), CCR2(Clone: SA203G11), BrdU(Clone: Bu20a), F4/80 (Clone: BM8), MerTK (Clone: 2B10C42 ), CD11a (Clone: I21/7), CD102 (Clone: 3C4), CD106 (Clone: 429), and I-A/I/E (Clone: M5/114.15.2) BioLegend (San Diego, CA). Intracellular staining was performed by fixing cells in Paraformaldehyde-based Fixation buffer (BioLegend) followed by permeabilization with Perm/Wash buffer (BioLegend). Cells were analyzed on a BD LSR II cytometer (BD Biosciences, San Jose, CA, USA) using FlowJo software (Tree Star, Ashland, OR, USA).

LPS stimulation assay: BMDM were primed with 150U/mL interferon- $\gamma$ (IFN $\gamma$ ) for 6 hours followed by LPS at final concentration of 10ng/mL (Sigma-Aldrich, St. Louis, MO) added into culture medium for 20 hours. Brefeldin A $5 \mu \mathrm{g} / \mathrm{mL}$ (BD Pharmingen, Bedford, MA) was added 4 hours before cell harvest and flow cytometry.

Phagocytosis Assay: The pHrodo ${ }^{\mathrm{TM}}$ Red BioParticles® Conjugates for Phargocytosis (P35364) (Molecular Probes, Eugene, OR) kit was used. Six days after cell plating with mCSF, and one day prior to the assay, BMDMs were recovered from culture and seeded. Cells were stimulated with IFN $\gamma$ for 4 hours and then culture medium was replaced with reconstituted red Zymosan A BioParticles. Cells were incubated at $37^{\circ} \mathrm{C}$ for 2 hours, trypsinized, and evaluated with flow cytometry.

Statistical analysis: Data were analyzed with GraphPad (Prism 2.8.1, San Diego, CA) using two-tailed unpaired t-test and ordinary one-way ANOVA with Dunnet's correction for multiple comparisons. Statistical significance was determined at $\mathrm{P}<0.05$.

Author contributions: FL designed experiments, acquired data and analyzed data; NC, CZ analyzed data; DGV wrote and reviewed the manuscript; JC reviewed the manuscript; EIP designed experiments, analyzed data, and wrote the manuscript.

Acknowledgments: This work was supported by the Boston Keratoprosthesis Research Fund, Massachusetts Eye and Ear, the Eleanor and Miles Shore Fund, the Massachusetts Lions Eye Research Fund, an unrestricted grant to the Department of Ophthalmology, Harvard Medical School, from Research to Prevent Blindness, NY, NY, NIH National Eye Institute core grant P30EY003790; Yeatts Family Foundation; Monte J Wallace Chair; Macula Society Research Grant award; an RPB Physician Scientist Award; NEI R21EY023079; NEI grant EY014104 (MEEI Core Grant); Loeffler Family fund; R01EY025362-01; ARI Young investigator Award. PLX5622 was kindly provided by Plexxikon Inc. 


\section{Figure Legends}

Fig. 1. CSF1R inhibition by PLX5622 affects the myeloid and lymphoid compartments of the bone marrow, spleen and blood.

Flow cytometric analysis of bone marrow cells isolated from $\mathrm{CCR} 2^{+/ \mathrm{RFP}}: \mathrm{CX} 3 \mathrm{CR} 1^{+/ \mathrm{GFP}}$ mice immediately, after 3-week treatment with CSF1R inhibitor (PLX5622), and at different timepoints after cessation of the CSF1R inhibitor. (A - F) CSF1R inhibition suppresses CCR2 ${ }^{+}$, $\mathrm{CX} 3 \mathrm{CR} 1^{+}, \mathrm{CD} 117^{+}$, and $\mathrm{CD} 34^{+}$cells. One week after cessation of CSF1R inhibitor, only macrophages recover in number, although with a lower expression of CX3CR1. (G) CSF1R inhibition does not affect $\mathrm{CD} 45^{+}, \mathrm{CD} 11 \mathrm{~b}^{+}$and $\mathrm{Ly}_{6 \mathrm{C}^{+}}$bone marrow myeloid cell populations, but does suppress $\mathrm{CD} 11 \mathrm{c}^{+}$dendritic cells, $\mathrm{CD} 4^{+}$and $\mathrm{CD}^{+} \mathrm{T}$ lymphocytes, as well as, CD115 ${ }^{+}$, $\mathrm{CD} 117^{+}$and $\mathrm{CD} 34^{+}$hematopoietic subsets, and upregulates $\mathrm{CD} 19^{+} \mathrm{B}$ cells. Three weeks after cessation of CSF1R inhibition, $\mathrm{CX} 3 \mathrm{CR} 1^{+}, \mathrm{CCR}^{+}, \mathrm{Ly}_{6 \mathrm{C}^{+}} \mathrm{CD}^{+}$and $\mathrm{CD}^{+}$sub-populations rebound; $\mathrm{Ly}_{6 \mathrm{G}^{+}}$granulocytes, $\mathrm{CD} 115^{+}$, and $\mathrm{CD} 117^{+}$cells remain suppressed; $\mathrm{CD} 4^{+} \mathrm{T}$ cells and $\mathrm{CD} 34^{+}$cells recover; and $\mathrm{CD} 19^{+} \mathrm{B}$ cells remain upregulated. $(\mathbf{H})$ Effects of CSF1R inhibition on the spleen's myeloid and lymphoid populations. Only CD19 ${ }^{+}$B cells remain unaffected. (I) CSF1R inhibition causes immediate suppression in the myeloid compartment late suppression of the lymphoid compartment of the blood. $n=5$ per group, mean $\pm \mathrm{SD}$, One-way analysis of variance with Dunnett's correction for multiple comparisons, $* P<0.05$, ** $P<0.01$, *** $P<0.001$, **** $P<0.0001$.

Fig. 2. CSF1R inhibition by PLX5622 affects the function and survival of tissue resident macrophages of the bone marrow, lung, peritoneum, spleen and liver.

(A-D) ex vivo evaluation of the function of bone marrow-derived macrophage (BMDM) from $\mathrm{CX} 3 \mathrm{CR} 1^{+/ \mathrm{GFP}}$ mice 3 weeks after cessation of CSF1R inhibitor. Macrophages from the bone marrow or spleen exhibit reduced proliferation 3 weeks after cessation of CSF1R inhibition. (EL) CSF1R inhibition suppresses IL-1 $\beta$, CD68 expression and phagocytosis of bone marrowderived macrophage following exposure to lipopolysaccharide, but does not affect their CD206 expression. (H) Schematic representation of the phagocytosis assay. (M, N) CSF1R inhibition causes long-term suppression of CD115 macrophage marker. (O) CSF1R inhibition reduces the number of tissue resident $\mathrm{CX} 3 \mathrm{CR} 1^{+}$femur, CD1 1a ${ }^{+}$lung, $\mathrm{CD} 102^{+}$peritoneum, $\mathrm{MHC}-\mathrm{II}^{\mathrm{h} / \mathrm{lo}}$ liver, but not $\mathrm{CD}_{106^{+}}$spleen macrophages, while consistently suppresses bone marrow-derived $\mathrm{CX} 3 \mathrm{CR} 1^{+/ \text {EGFP }}$ macrophages in these tissues. $\mathrm{n}=5$ per group, mean $\pm \mathrm{SD}$, Independent $\mathrm{t}$-test, $* P<0.05, * * P<0.01, * * * P<0.001, * * * * P<0.0001$. 


\section{Bibliography}

1. M. R. P. Elmore et al., Colony-stimulating factor 1 receptor signaling is necessary for microglia viability, unmasking a microglia progenitor cell in the adult brain. Neuron 82, 380-397 (2014).

2. S. Mok et al., Inhibition of CSF-1 Receptor Improves the Antitumor Efficacy of Adoptive Cell Transfer Immunotherapy. Cancer research 74, 153-161 (2014).

3. A. M. Hilla, H. Diekmann, D. Fischer, Microglia Are Irrelevant for Neuronal

Degeneration and Axon Regeneration after Acute Injury. Journal of Neuroscience 37, 6113-6124 (2017).

4. V. Bellver-Landete et al., Microglia are an essential component of the neuroprotective scar that forms after spinal cord injury. Nature communications 10, 518 (2019).

5. J. Bonnardel et al., Stellate Cells, Hepatocytes, and Endothelial Cells Imprint the Kupffer Cell Identity on Monocytes Colonizing the Liver Macrophage Niche. Immunity 51, 638-654 e639 (2019).

6. J. A. Santamaria-Barria et al., Csf1r or Mer inhibition delays liver regeneration via suppression of Kupffer cells. PLoS One 14, e0216275 (2019).

7. T. A. Evans et al., High-resolution intravital imaging reveals that blood-derived macrophages but not resident microglia facilitate secondary axonal dieback in traumatic spinal cord injury. Experimental neurology 254, 109-120 (2014).

8. T. A. Wynn, K. M. Vannella, Macrophages in Tissue Repair, Regeneration, and Fibrosis. Immunity 44, 450-462 (2016).

9. S. J. Karlen et al., Monocyte infiltration rather than microglia proliferation dominates the early immune response to rapid photoreceptor degeneration. Journal of neuroinflammation 15, a020545-020516 (2018).

10. E. I. Paschalis et al., Permanent neuroglial remodeling of the retina following infiltration of CSF1R inhibition-resistant peripheral monocytes. Proceedings of the National Academy of Sciences of the United States of America 115, E11359-E11368 (2018).

11. E. I. Paschalis et al., The Role of Microglia and Peripheral Monocytes in Retinal Damage after Corneal Chemical Injury. The American journal of pathology 188, 1580-1596 (2018).

12. E. I. Paschalis et al., Microglia Regulate Neuroglia Remodeling in Various Ocular and Retinal Injuries. Journal of immunology (Baltimore, Md. : 1950) 202, 539-549 (2019).

13. N. N. Dagher et al., Colony-stimulating factor 1 receptor inhibition prevents microglial plaque association and improves cognition in 3xTg-AD mice. Journal of neuroinflammation $\mathbf{1 2}$, 139 (2015).

14. D. Kokona, A. Ebneter, P. Escher, M. S. Zinkernagel, Colony-stimulating factor 1 receptor inhibition prevents disruption of the blood-retina barrier during chronic inflammation. $J$ Neuroinflammation 15, 340 (2018).

15. S. K. Halder, R. Milner, A critical role for microglia in maintaining vascular integrity in the hypoxic spinal cord. Proc Natl Acad Sci U S A 116, 26029-26037 (2019).

16. J. M. Hutchinson, L. G. Isaacson, Elimination of microglia in mouse spinal cord alters the retrograde CNS plasticity observed following peripheral axon injury. Brain Res 1721, 146328 (2019).

17. Y. Okunuki et al., Retinal microglia initiate neuroinflammation in ocular autoimmunity. Proceedings of the National Academy of Sciences 156, $201820387-201820310$ (2019).

18. E. L. Gautier et al., Gene-expression profiles and transcriptional regulatory pathways that underlie the identity and diversity of mouse tissue macrophages. Nature Immunology 13, 11181128 (2012). 
bioRxiv preprint doi: https://doi.org/10.1101/2019.12.27.889469; this version posted June 16, 2020. The copyright holder for this preprint (which was not certified by peer review) is the author/funder, who has granted bioRxiv a license to display the preprint in perpetuity. It is made available under aCC-BY-ND 4.0 International license.

19. S. Chakarov et al., Two distinct interstitial macrophage populations coexist across tissues in specific subtissular niches. Science 363 (2019). 
bioRxiv preprint doi: https://doi.org/10.1101/2019.12.27.889469; this version posted Jupe 16, 2020. The copyright holdeffor this preprint

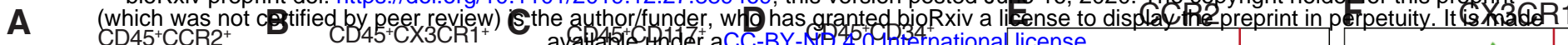
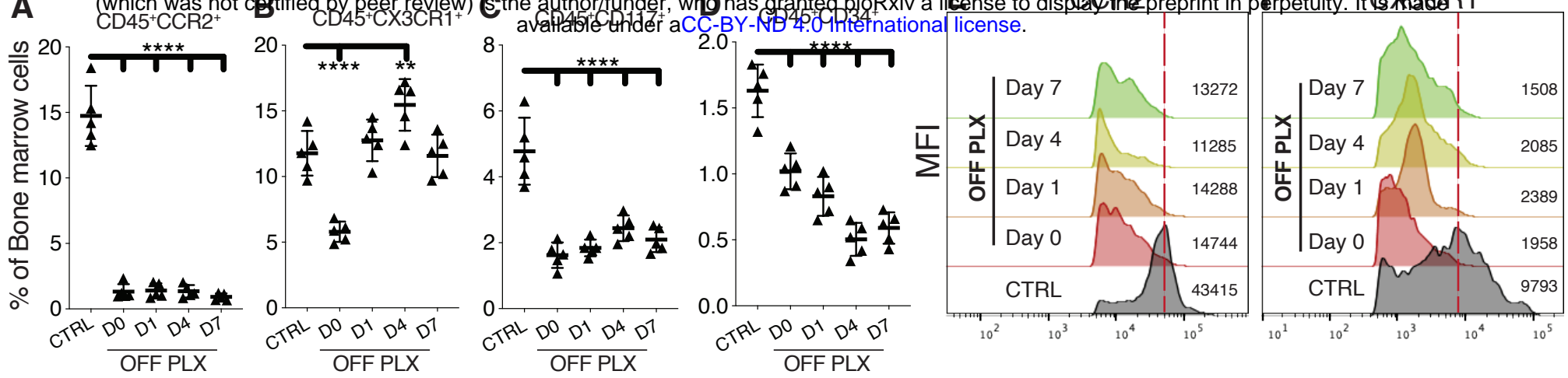

\section{G \\ Bone Marrow control, on PLX and 3 weeks OFF PLX}
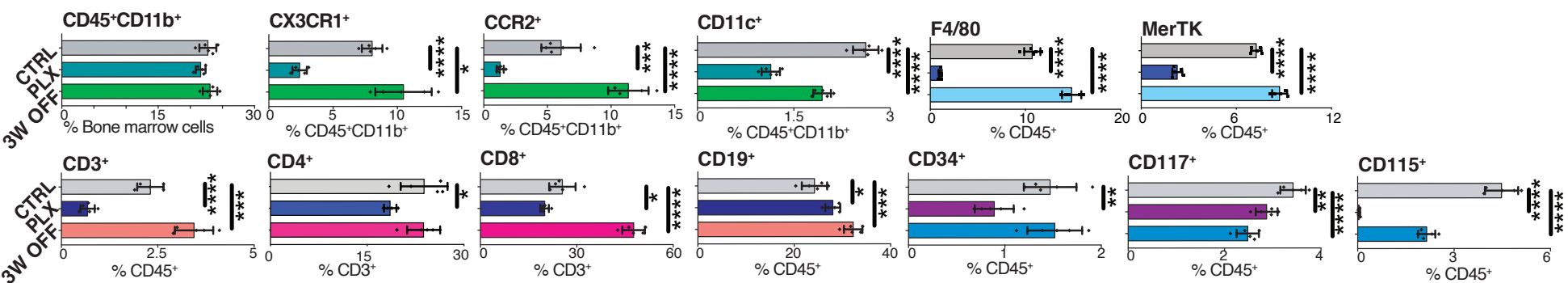

\section{Spleen control, on PLX and 3 weeks OFF PLX}

H

CX3CR1+

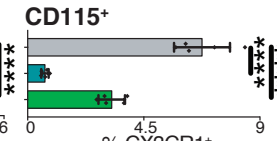

CD19+

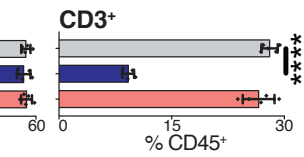

$\mathrm{CD}^{+}$
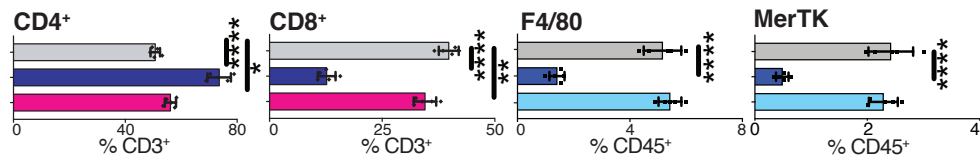

Blood control, on PLX and 3 weeks OFF PLX
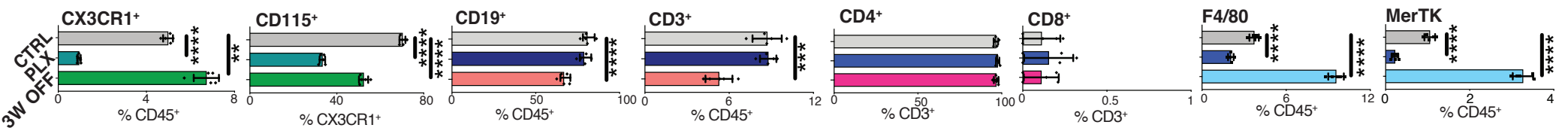
a

A Bone Marrow CX3CR1+
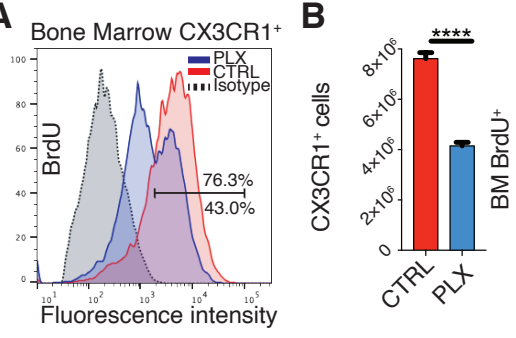

C Spleen $\mathrm{CX} 3 \mathrm{CR}^{+}$
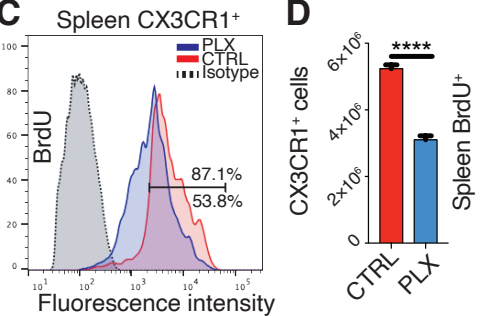

E

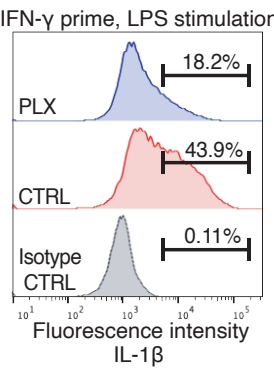

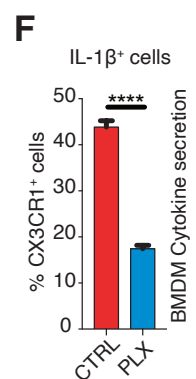

G

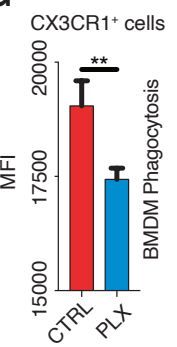

PLX5622 3 weeks

$\mathbf{H}$

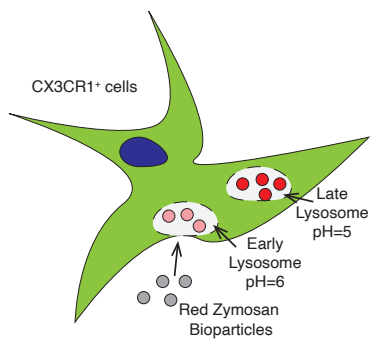

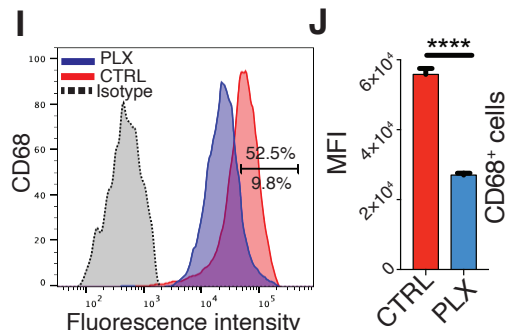
K

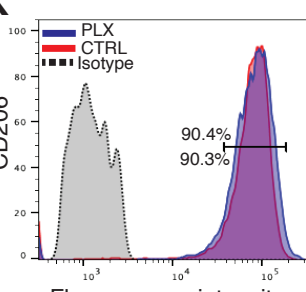

L

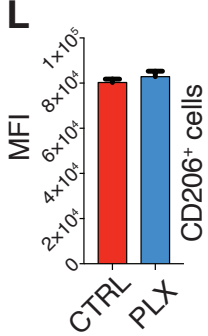

CD115 expression in BMDM
M

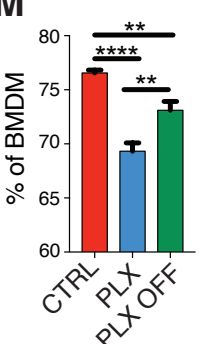

$\mathbf{N}$

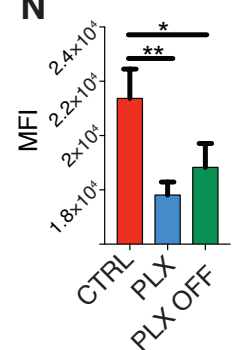

0

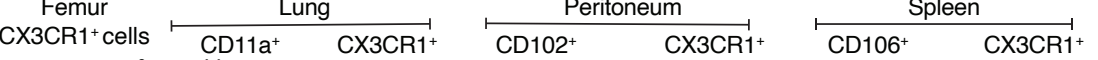
C57BL/6 BMT CX3CR1+EGFP Analysis 208 -PLX5622 3WK
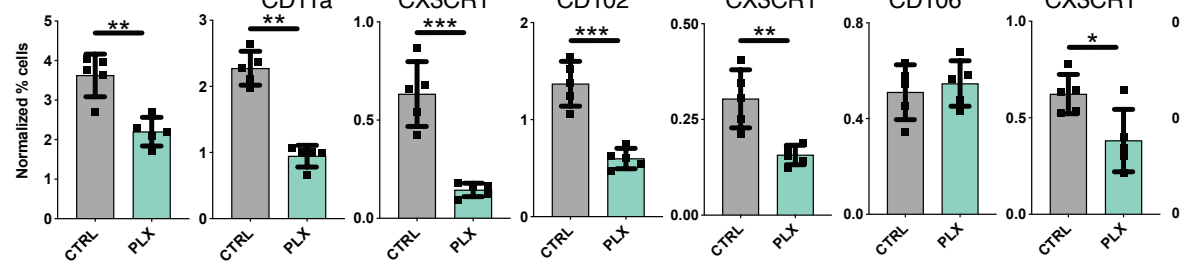

MHC $\|^{\text {hi }} \quad$ MHC II CX3CR $1^{+}$

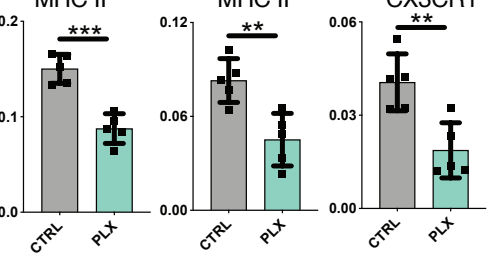

